\title{
Chapter
}

\section{NEOTISSUE REMODELING OF TISSUE-ENGINEERED ARTERIAL GRAFT}

Shuhei Tara ${ }^{1,2}$, Toshihiro Shoji ${ }^{*}$, and Toshiharu Shinoka ${ }^{1,2}$

1 Tissue Engineering Program, Nationwide Children's Hospital, Columbus, OH, USA

${ }^{2}$ Department of Cardiothoracic Surgery, The Heart Center, Nationwide Children's Hospital, Columbus, OH, USA 


\section{Contents}

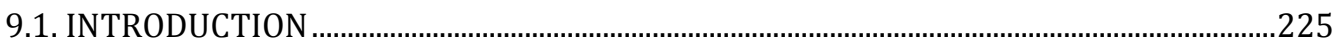

9.1.1. Neoartery components as a basis for TEVG remodeling .......................................226

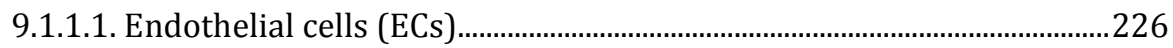

9.1.1.2. Smooth muscle cells (SMCs) .....................................................................227

9.1.1.3. Extracellular matrix (ECM) ………………………………........................22

9.1.2. Tissue remodeling process in arterial TEVG...........................................................228

9.1.3. Calcific deposition......................................................................................................230

9.2. PUTATIVE MECHANISMS OF NEOTISSUE FORMATION IN TEVG REMODELING .......231

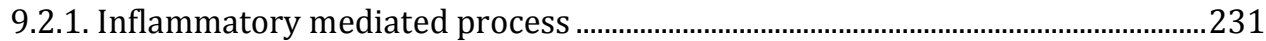

9.2.2. Endothelial-to-mesenchymal transition ....................................................................23

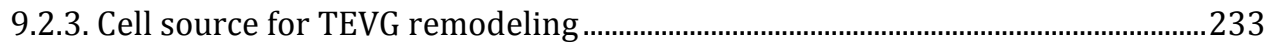

9.2.3.1. Adjacent blood vessel (Transanastomotic outgrowth) ..........................234

9.2.3.2. Transmural ingrowth ..............................................................................2.

9.2.3.3. Migration from circulating blood stream.....................................................234

9.3. BIODEGRADABLE POLYMERS FOR ARTERIAL TEVGs.......................................................235

9.3.1. Structural characteristics of arterial TEVGs .........................................................236

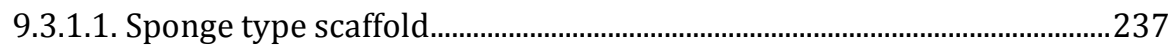

9.3.1.2. Electrospinning technique ........................................................................237

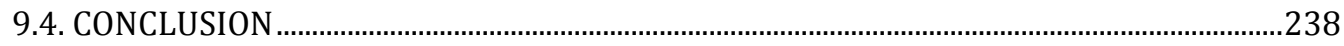

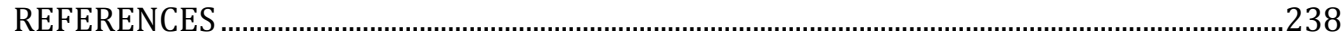




\subsection{INTRODUCTION}

Atherosclerotic cardiovascular diseases (CVDs), a systemic narrowing and hardening of arteries, include conditions such as coronary arterial disease, carotid artery stenosis, and peripheral arterial disease. CVDs are a leading cause of death or impaired quality of life for millions of individuals in developed nations. The most common corrective procedure for CVDs is surgical intervention using autologous arterial and/or venous grafts. However, the use of autologous grafts leads to prolonged operative times and increased risk of peri-operative infection. Additionally, many patients lack suitable donor tissue, either as a result of their underlying vascular disease or previous surgery. Synthetic materials such as expanded poly(tetrafluoroethylene) (ePTFE, Goretex ${ }^{\circledR}$ ) and poly(ethylene terephthalate) (PET, Dacron ${ }^{\circledR}$ ) provide an alternative strategy, but when applied to small-diameter $(<6 \mathrm{~mm})$ arterial grafts, this approach has limited efficacy due to various complications such as progressive stenosis, thrombosis, calcium deposition, host rejection, increased risk of infection, and the persistent need for anticoagulation therapy.

The purpose of tissue engineering is to develop alternative materials that integrate with the patient's native tissue to restore physiologic function [1]. This often involves the use of biodegradable synthetic materials, termed scaffolds, to provide a three-dimensional surface for cellular proliferation and neotissue formation during neovessel remodeling. In addition, the traditional concept of tissue engineering consists of the isolation and use of cells or cell substitutes, and the integration of the isolated cells and scaffold via a seeding technique [2]. In 1986, Weinberg and Bell produced what was widely regarded as the first tissue-engineered blood vessel substitute. It consisted of bovine endothelial cell (EC), smooth muscle cell (SMC) and fibroblast cell cultures embedded in a collagen gel [3]. However, this tissue-engineered construct lacked adequate strength and required reinforcement with a Dacron ${ }^{\circledR}$ mesh. In following years, vascular grafts were improved, refined, and ultimately reached clinical application in 1999 [4]. The first human clinical trial investigating tissue engineered vascular graft (TEVG) implantation in children with congenital heart defects began in 2001. The highly porous biodegradable polymeric scaffold was comprised of poly(L-lactide-co- $\varepsilon$-caprolactone) (PLCL) reinforced by poly(glycolic acid) mesh [5] and seeded with bone marrow derived mononuclear cells (BM-MNCs). The application of TEVGs in a lowpressure environment for patients with congenital heart disease has several advantages such as growth potential, favorable biocompatibility, and low risk of infection or rejection. Clinical evidence has now established that TEVGs are safe and effective to use in pediatric patients undergoing extracardiac total cavopulmonary connection procedures [5-7]. However, to apply TEVGs to an arterial system, a scaffold must be able to withstand mechanical loadings akin to the native artery such as arterial pressure, shear stresses, durability, and 
compliance. With this in mind, the efficacy of various TEVG types, with or without cell seeding, for small diameter arterial grafts has been demonstrated in animal models [8-11].

A biodegradable vascular graft is completely reconstituted by host-derived cells over the course of its inflammation-mediated process [12]. It was previously believed that the progenitor cells in BM-MNCs differentiated into mature vascular cells in the developing TEVG. In fact, small populations of hematopoietic and vascular progenitor cells were identified within seeded BM-MNC populations. However, the number of seeded cells in the graft decreased rapidly within the first few days after implantation, ultimately resulting in the absence of all BM-MNCs within one-week, leaving many questions as to the mechanism of TEVG neotissue formation [12]. Even though the complete mechanism of neotissue formation during graft-material absorption remains to be fully elucidated, multiple TEVG remodeling processes have been proposed. In this chapter, we introduce the remodeling process and putative mechanisms of neotissue formation in arterial TEVGs, and lastly feature the differences in scaffold materials and constructions to fabricate an ideal arterial TEVG.

\subsubsection{Neoartery components as a basis for TEVG remodeling}

\subsubsection{Endothelial cells (ECs)}

ECs possess various physiologic functions in vivo, such as the transport of blood constituents, thromboresistance, angiogenesis induction, and blood pressure control via vasoconstriction/vasodilation. Additionally, ECs are able to prevent neointimal hyperplasia through the inhibition of bioactive substances responsible for extracellular matrix (ECM) production, and smooth muscle cell (SMC) migration and proliferation.

The development of a confluent endothelial monolayer is a vital and primary step for well-organized TEVG neotissue formation. However, ECs have limited capacity for regeneration and can no longer divide after about 70 cell cycles. In humans, currently available graft implants show limited EC ingrowth, typically not extending 1-2 $\mathrm{cm}$ beyond the anastomoses [13]. After implantation for peripheral artery disease, the number of ECs observed on the luminal surface of synthetic grafts was less than $10 \%$ of that seen in native vessels [14]. To improve graft surface endothelialization, EC seeding has been applied to synthetic arterial grafts. However the clinical efficacy of EC seeding to produce patent synthetic grafts remains a controversial topic [15]. As a result, the safekeeping of ECs from damaging factors, such as oxidative and sheer stresses, remains a major hurdle for the clinical application of arterial TEVGs. 


\subsubsection{Smooth muscle cells (SMCs)}

SMCs regulate vasocontraction and are an integral component of an efficient and stable blood vessel. SMCs are the predominant component of the ECM complex that ultimately defines blood vessel mechanical properties [3]. Yue et al. investigated the seeding of cultured SMCs onto biodegradable scaffolds implanted in rat aortas, and in comparison to unseeded controls, the implanted constructs demonstrated rapid neomedia formation [16]. Similarly, Niklason et al. developed a tissue-engineered graft by seeding SMCs onto a poly(glycolic acid) (PGA) scaffold that was then cultured in vitro in a pulsatile radial stress environment for 8 weeks [17]. The SMC-seeded PGA grafts demonstrated physiologic and mechanical functions comparable to native human vessels and are being considered for clinical application [18].

SMCs are complex cells capable of existing in heterogeneous populations and switching phenotypes when exposed to various stimuli (i.e. contractile to synthetic) [19]. A synthetic dedifferentiated SMC phenotype is characterized by its migration, proliferation, and ECM synthesis in the vascular wall. These dedifferentiated SMCs can be identified early on in the developing vasculature through $\alpha$-SMA expression detection [20]. In contrast, the contractile apparatus of differentiated SMCs have less proliferation potential and are distinguished by their expression of differentiated SMC markers such as smooth muscle-myosin heavy chain (SM-MHC) [20]. Although synthetic SMCs are necessary to obtain favorable ECM formation in neovessels during the neotissue remodeling process, excessive synthetic SMCs may also induce TEVG stenosis causing neotissue hyperplasia.

\subsubsection{Extracellular matrix (ECM)}

As the scaffold degrades, the ECM undergoes continuous remodeling. The ECM is predominantly comprised of elastin, proteoglycans, and collagen type I and III. ECM mechanical properties that are relevant to arterial vessel function includes tensile stiffness, compressibility, and elasticity. Collagen provides the ECM with the tensile strength to resist rupture and proteoglycans contribute to ECM compressibility [21,22].

Elastin is the most important component of the ECM as it confers elasticity to arteries. It prevents dynamic tissue creep by stretching under load and recoils back to its original conformation after the load is released. Elastin is also a potent autocrine regulator of vascular SMC activity. Accordingly, elastin's regulatory effects are significant in preventing fibrocellular pathology [23]. Upon insult however, elastic matrices are difficult to repair due to: 1) poor elastin precursor (tropoelastin) synthesis by adult cells, 2) inefficient recruitment and crosslinking of tropoelastin into an elastic matrix, and 3) further organization into elastic fibers [23,24]. Hence, the resourceful utilization of elastic materials and tropoelastin coatings on scaffolds, may be helpful to augment arterial TEVG elastin functions. 


\subsubsection{Tissue remodeling process in arterial TEVG}

A better understanding of the tissue remodeling process is critical for the improvement, continued development, and rational design of arterial TEVGs. Neovessel formation is a dynamic process characterized by progressive scaffold degradation due to hydrolysis, cellular infiltration into scaffold, and ECM deposition. As the remodeling time course and process can be altered through the use of different materials and scaffold structures, the trade-off between scaffold degradation and neotissue formation must be considered when TEVGs are applied to the high pressures of arterial circulation.

During the acute phase of tissue remodeling (within 6 weeks post implantation), vascular neotissue of arterial TEVGs constructed with poly(L-lactic acid) (PLA) fibers coated with PLCL are characterized by: 1) collagen type I and III deposition, 2) a lack of elastin, 3) SMC proliferation in the medial layer, and 4) partial endothelialization in the internal scaffold lumen [8]. Within 4 months post-implantation, favorable endothelialization is achieved (Figure 1) [25], along with gradual increases in collagen deposition, SMC proliferation, and elastin deposition (Figure 2) [25].

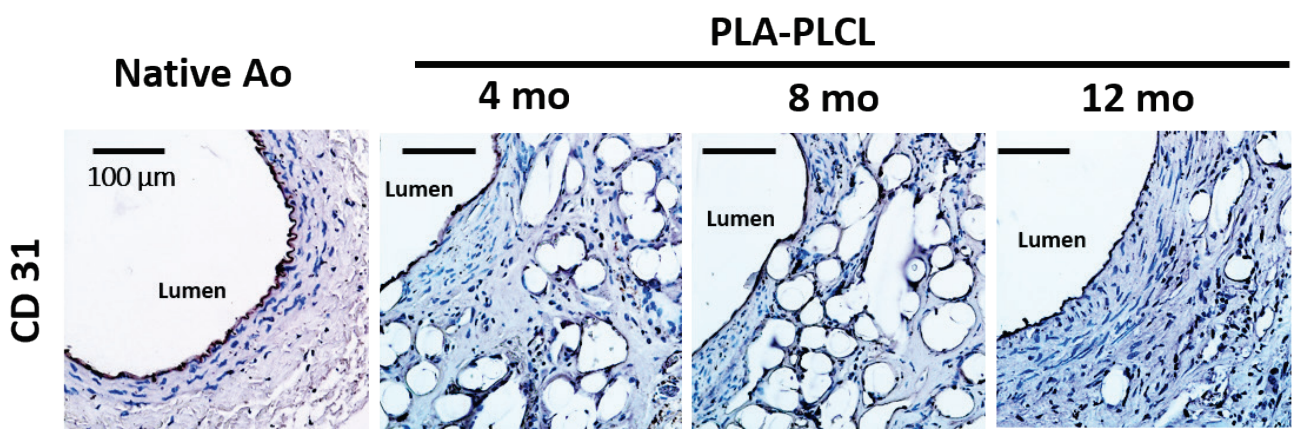

Figure 1. Endothelial cell identification with CD 31 immunohistochemical staining. Grafts at 4, 8, 12 months after implantation and native abdominal aortas of mice were explanted and analyzed [25]. 


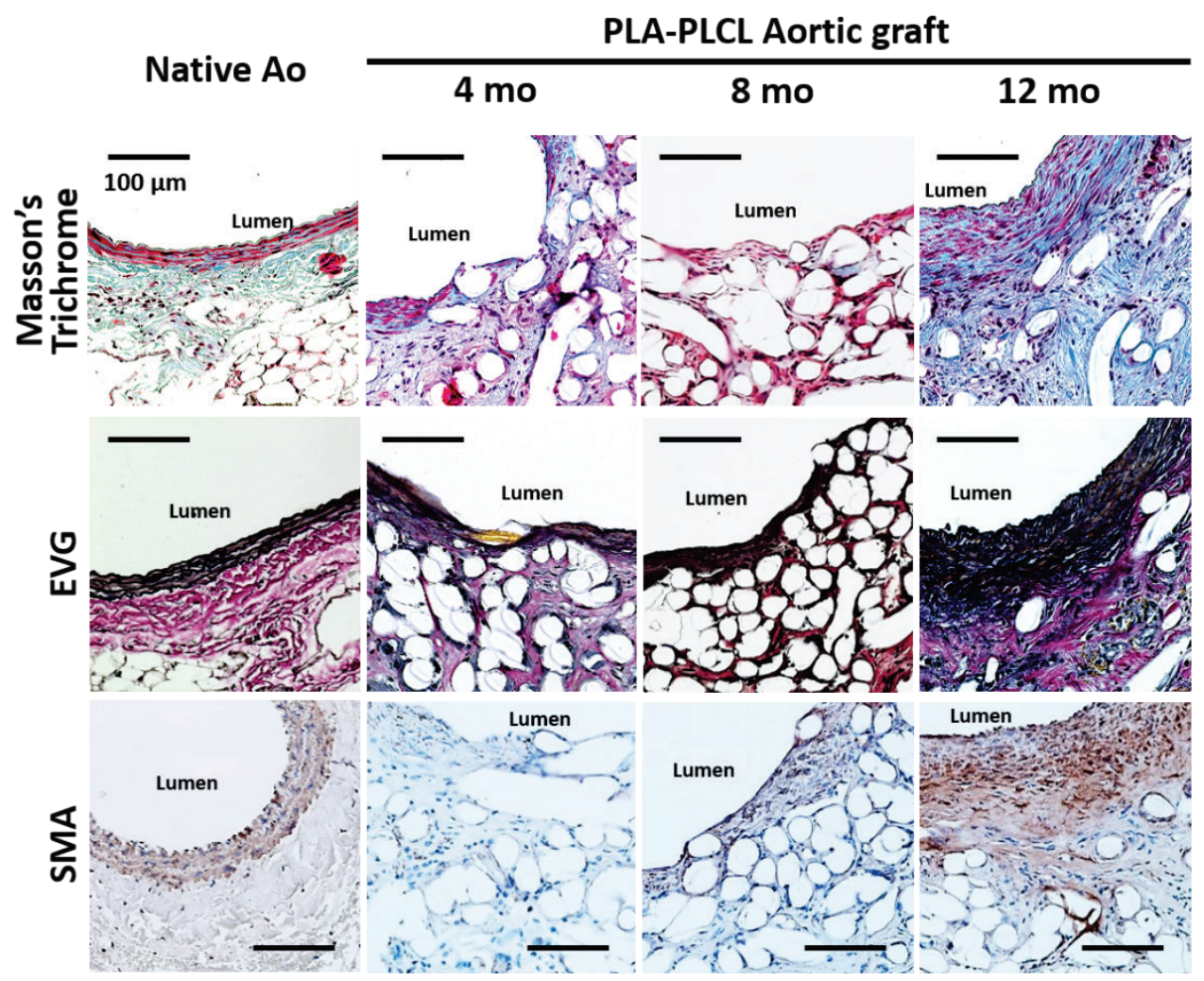

Figure 2. Representative histologic image of Masson's Trichrome staining for collagen deposition, Elastica van Gieson (EVG) staining for elastin formation, and immunohistochemical smooth muscle actin (SMA) staining for smooth muscle cells within the neointimal at 4, 8, 12 months after implantation into mouse abdominal aorta [25].

The primary cellular component during the early stages of arterial TEVG remodeling are matrix metallopeptidase 9 (MMP-9) promoted macrophages and monocytes [26]. MMP-9 is the most prominent MMP type present during foreign body inflammatory responses [27] and is shown in an acute phase of neotissue formation, peaking 1 week after TEVG implantation, and decreaseing thereafter. Moreover, SMC migration is dependent on both MMP-9 and -2 . MMP-2 is primarily produced by inflammatory cell stimulated mesenchymal cells and peaks during the late phase of neotissue formation. However, MMP activity in the arterial TEVG model, though similar to the venous model, is prolonged because of remaining fiber remnants.

TGF- $\beta$ is a multifunctional cytokine that regulates cell adhesion, migration, differentiation, proliferation and apoptosis. TGF- $\beta$ signaling plays an essential role in vascular remodeling and disruptions to its signaling pathway is known to cause vascular dysfunctions such as aortic aneurysms. After short-term 
(6 week) follow-up, PLA-PLCL aortic grafts possessed adequate mechanical properties and strength to function as arterial grafts and displayed no signs of aneurysmal change [8]. However, many grafts formed aneurysmal changes or ruptured later (12 months) with TGF- $\beta$ elevation [25]. Thus, continuous TGF- $\beta$ elevation may induce vascular dysfunction of arterial TEVGs followed by subsequent aneurysmal changes. Of further interest, angiotensin receptor blockers are known to inhibit TGF- $\beta$ signaling and prevent aortic aneurysm in Marfan syndrome [28,29]. Consequently, angiotensin receptor blockers have the therapeutic potential to prevent aneurysmal changes in arterial TEVGs.

\subsubsection{Calcific deposition}

Implants are susceptible to calcification during neovessel remodeling, a potentially fatal longer term complication. However, the role that the physical scaffold structure has on long-term calcific deposition in arterial TEVG neotissue remains a field yet to be studied.

Chondroid metaplasia takes place in intimal hyperplasia layers and contributes to arterial TEVG calcification [30]. Although the precise mechanisms underlying osteogenic differentiation of vascular cells during atherosclerosis remain undefined, vascular SMCs can undergo osteogenic differentiation and calcification [31,32]. However, normal vascular SMCs have the potential to prevent calcium deposition as they express transcription factors of both osteogenesis and osteoclastogenesis. Well organized neointima, abundant with SMCs, better prevents calcific deposition in comparison to thin neointima on small-pore nanofiber grafts (Figure 3) [33]. Therefore, a degree of neointimal SMCs is required to eliminate calcium deposition and sustain the balance of osteogenesis and osteoclastogenesis. 

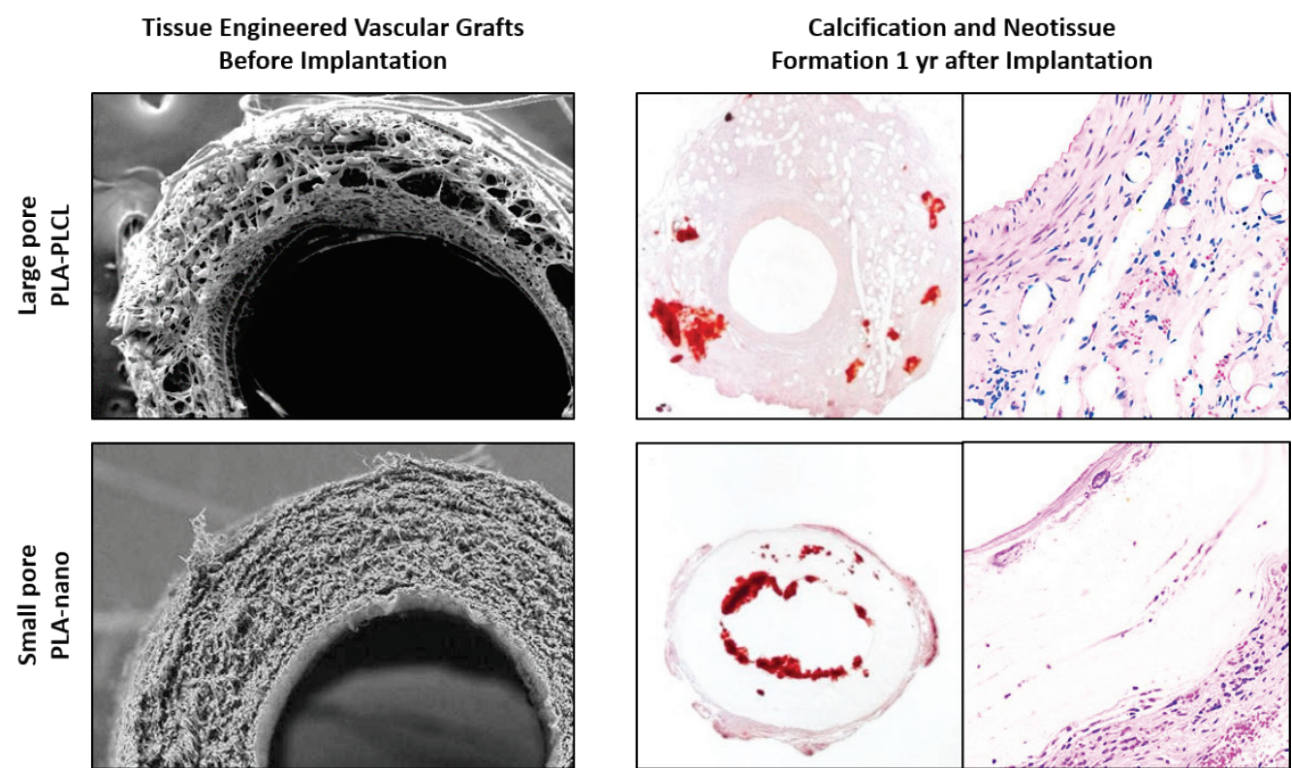

Figure 3. Large-pore PLA-PLCL grafts created a well-organized neointima and prevented calcific deposition compared to small-pore, electrospun PLA-nanofiber grafts [33].

The scaffold degradation period is another key aspect to consider calcification in tissue remodeling for biodegradable vascular grafts [10]. At one year follow up, in a rapid remodeling process, fast degrading elastomers developed into well-organized neotissue without calcification [34]. In contrast, high density (small pore size) nanofiber fabric have longer polymer degradation periods and lack transmural cellular migration onto the scaffold [33]. Consequently, the combined effects of small pore size and slow degradation, results in calcified deposition in neotissue remodeling and prolonged foreign body reactions.

\subsection{PUTATIVE MECHANISMS OF NEOTISSUE FORMATION IN TEVG REMODELING}

Although, the precise mechanism of neotissue formation in TEVG remodeling remains to be fully elucidated, molecular and cellular mechanisms underlying TEVG remodeling and vascular neotissue formation have been proposed.

\subsubsection{Inflammatory mediated process}

Monocyte and macrophage mediated inflammation plays a crucial role in vascular neotissue formation (Figure 4), as well as the development of stenosis in a scaffold [12]. Clodronate is known to deplete monocytes and macrophages 
in vivo. Liposomal clodronate was administered into a mouse model to evaluate the function of monocytes and macrophages, and the study demonstrated the importance of monocytes/macrophages in TEVG tissue remodeling as clodronate administration effectively suppressed neotissue formation and vascular repair [35]. On the other hand, excessive macrophage infiltration produces neotissue hyperplasia, which leads to stenotic/occluded grafts.
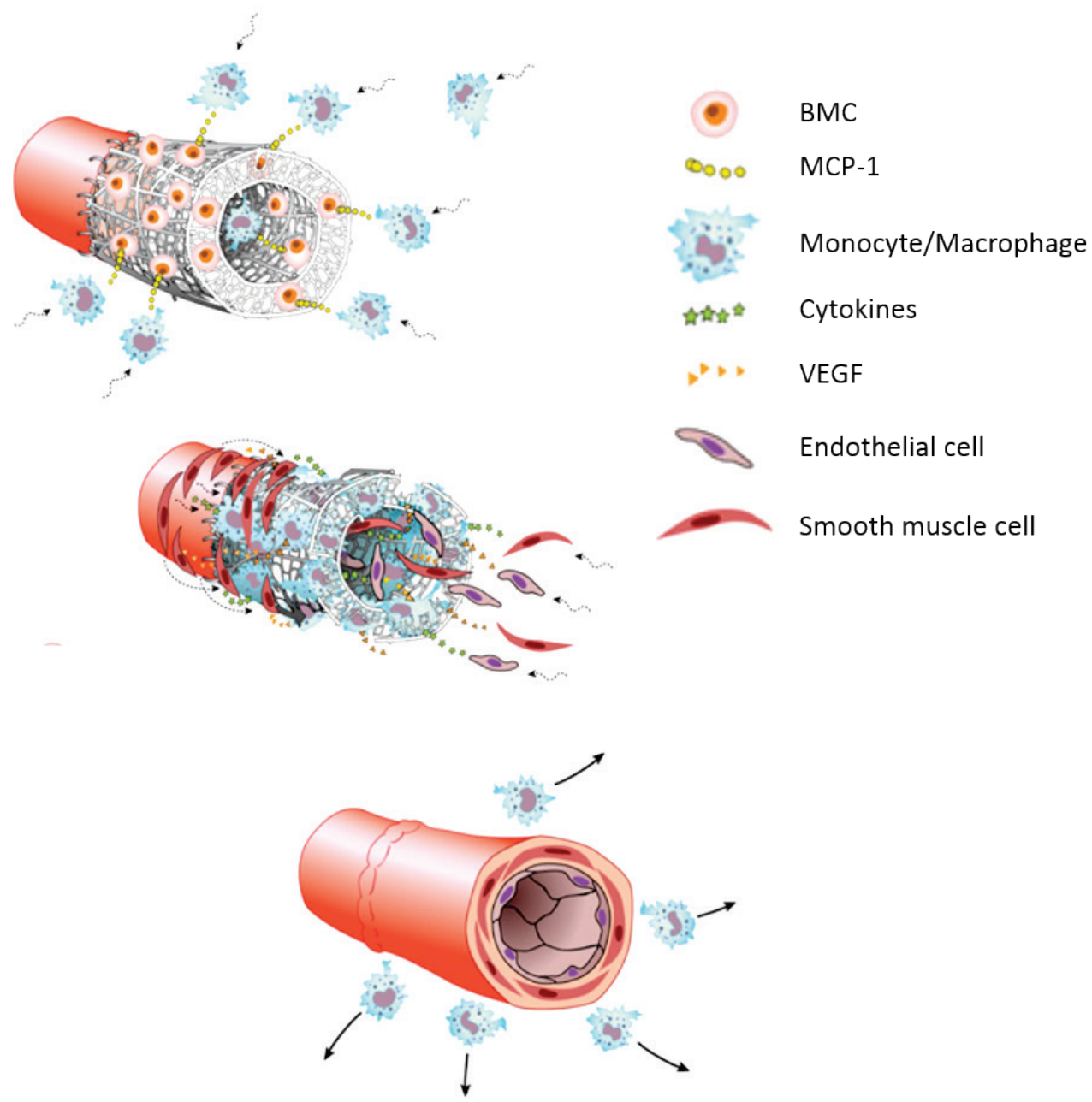

Figure 4. Proposed mechanism of neovessel formation after implantation of a cell-seeded biodegradable scaffold. Early pulse of monocyte chemoattractant protein-1 (MCP-1) and related cytokines from seeded bone marrow-derived mononuclear cells

(BM-MNCs) enhances early monocyte recruitment to the scaffold. Infiltrating monocytes release multiple angiogenic cytokines and growth factors (e.g., VEGF), which recruit smooth muscle cells and endothelial cells [12]. 
Macrophages in the scaffold release a variety of chemokines, cytokines, and growth factors. Macrophage-derived signaling molecules induce migration of adjacent host SMCs and ECs into the scaffold. As scaffold degradation begins, macrophages, fibroblasts, and SMCs start to deposit ECM, which continues to undergo remodeling throughout the formation of a viable TEVG. Given sufficient time, the biomechanical properties of the neovessel are no longer determined by scaffold characteristics, but are instead characterized by its collagen and elastin content [36]. As a final result, the TEVG has completely transformed into a neovessel, with cellular, biomechanical, and physiologic characteristics similar to that of a native vessel.

\subsubsection{Endothelial-to-mesenchymal transition}

The classical mechanism for cell differentiation is for progenitor cells to transform from immature to mature cell types via a well-ordered pathway. On the other hand, the endothelial-to-mesenchymal transition (EndMT) is a complex process, where endothelial cells lose their cellular polarity and adhesion abilities, while gaining migratory and invasive properties as they develop into mesenchymal cells, including SMC-like and fibroblast-like cells [37]. The EndMT has been shown to occur in wound healing, organ fibrosis, and also contributes to postnatal processes, such as promoting vein graft remodeling [38]. The EndMT contribution to vascular neotissue and stenosis formation have been demonstrated during TEVG remodeling [39]. TGF- $\beta$ is thought to drive EndMT in both a small mothers against decapentaplegic (SMAD)-dependent and independent manner $[40,41]$. To this end, anti-TGF- $\beta$ therapy has been pointed to for its potential utility to prevent TEVG stenosis.

\subsubsection{Cell source for TEVG remodeling}

In contrast to previous suggestions, neotissue cells are not derived from seeded cells [42]. Instead, several sources have been proposed for the spontaneous in vivo endothelialization and SMC proliferation in TEVGs. Such proposals include: 1) the transanastomotic outgrowth from the adjacent native blood vessel, 2) transmural ingrowth of capillaries from the adventitial side of the vascular graft, and 3) the migration of circulating endothelial progenitor cells from the luminal surface of vascular grafts (Figure 5). 


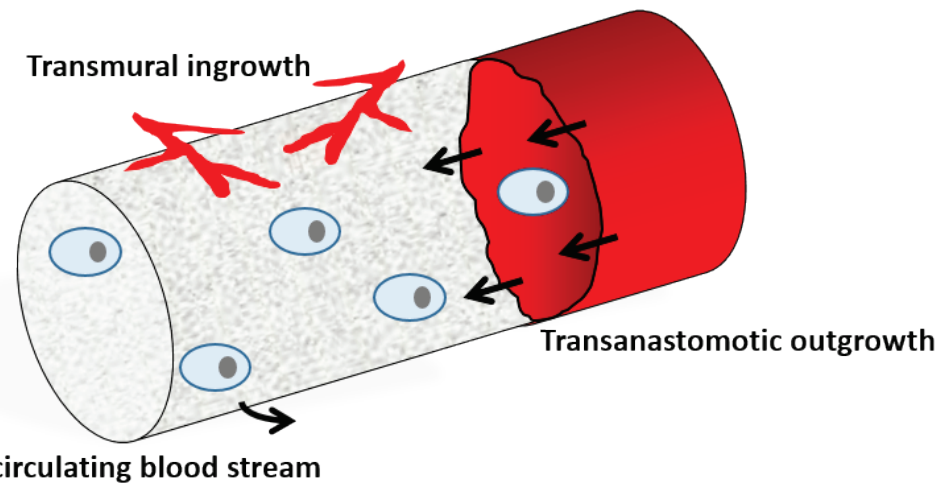

Migration from circulating blood stream

Figure 5. Schematic image of cell source for TEVG remodeling. Three different sources have been proposed for the spontaneous in vivo endothelialization and SMC proliferation in TEVGs.

\subsubsection{Adjacent blood vessel (Transanastomotic outgrowth)}

Transanastomotic outgrowth from the adjacent blood vessel is the principal source of SMCs and ECs in implanted TEVGs [43]. After implantation however, transanastomotic outgrowth slows down gradually [44], and typically does not extend 1-2 cm beyond the anastomoses [13]. Additionally, transanastomotic intimal hyperplasia is a major contributor to graft failure. Transanastomotic outgrowth provides sufficient endothelial coverage for short vascular grafts in small animal models, however other cells sources will be necessary to obtain favorable SMC proliferation and endothelialization in longer grafts.

\subsubsection{Transmural ingrowth}

Transmural endothelium ingrowth was clearly distinguished experimentally from transanastomotic outgrowth by using a unique looped interposition graft model and provides another realistic mechanism of spontaneous in vivo endothelialization [44]. Capillaries can penetrate the graft wall and provide sources of SMCs and luminal endothelium, where the aforementioned cells can adopt large vessel phenotypes. Moreover, capillaries can prove rapid coverage of porous synthetic graft surfaces that are in contact with the arterial circulation [14]. Of note however, is that tightly knit, electrospun nanofiber by itself, or used as a scaffold sheath to provide mechanical strength, may restrict transmural capillary ingrowth and/or cell migration from outside the graft.

\subsubsection{Migration from circulating blood stream}

$\mathrm{CD} 4^{+}$endothelial progenitor cells (EPCs) exist in circulating blood and can differentiate into mature ECs [45]. After mobilization from bone marrow, CD34+ EPCs participate in postnatal neovascularization [46]. After seeding onto decellularized xenogeneic arterial grafts, ex vivo expanded EPCs isolated 
from peripheral sheep blood, effectively achieved luminal coverage. This indicates EPCs possess the ability to endothelialize TEVGs [47]. Additionally, bone marrow cells may have potential to give rise to other vascular progenitor cells that contribute to vascular remodeling [48]. To increase EPC and smooth muscle progenitor cell migration from the peripheral blood onto the luminal surface, a stromal cell-derived factor- $1 \alpha$ /heparin coating was applied to a TEVG. The coated TEVG resulted in accelerated endothelialization and SMC differentiation [49].

\subsection{BIODEGRADABLE POLYMERS FOR ARTERIAL TEVGs}

The role of a scaffold is to provide a temporary three-dimensional structure for cellular attachment, infiltration, and proliferation. Scaffold materials should be highly porous and possess biomimetic properties, thereby facilitating cellular infiltration, neotissue formation, and integration with native tissue [50]. Biodegradable polymers utilized in arterial scaffolds are well suited to fulfill these requirements (Figure 6).

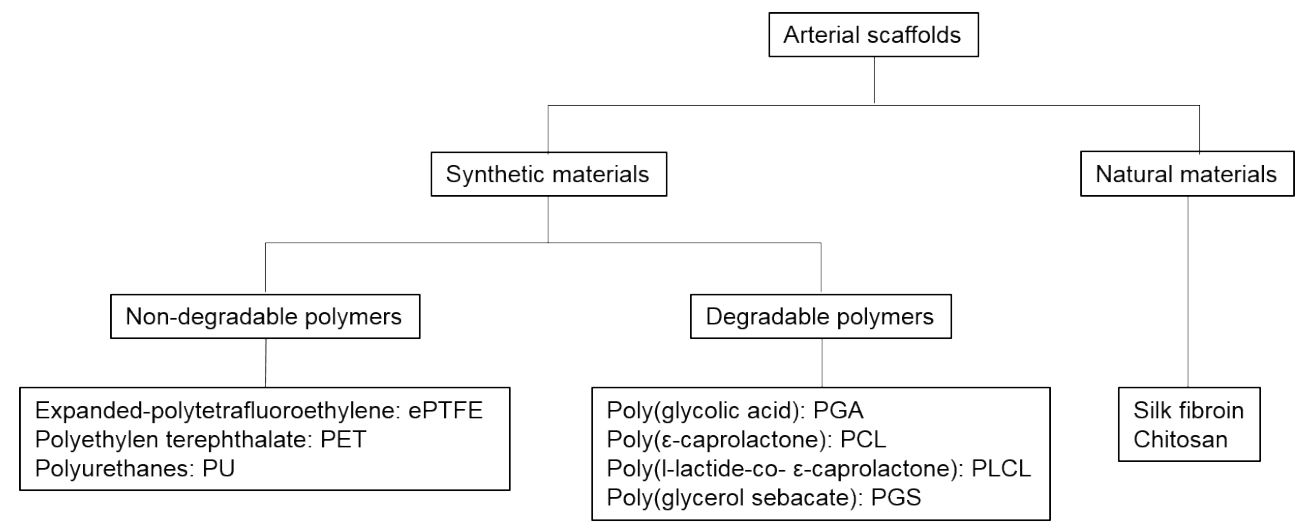

Figure 6. Flowchart for arterial scaffold. Both synthetic and natural materials are applied to arterial TEVGs as a biodegradable polymer.

Biodegradable polymers serve as temporary blood vessel scaffolds before being replaced by ingrowing tissue. Scaffold degradation first exhibits loss of mechanical properties, followed by a decrease in mass/volume. The degradation rate of biodegradable polymers depends on its initial molecular weight, exposed surface area, and crystallinity. The ideal scaffold for vascular applications meet the following criteria: 1) provides a suitable surface for cell adhesion and proliferation, 2) is highly porous to allow for neotissue growth and the exchange of nutrients and metabolic waste, and 3) is biodegradable, ultimately restoring physiologic function without reinforcement with 
non-degradable synthetic materials such as Goretex ${ }^{\circledR}$ and Dacron ${ }^{\circledR}$. The crucial first step in vascular engineering construct design is the selection of appropriate biodegradable scaffold materials and this is dependent on various factors, such as biocompatibility, biodegradability, and mechanical properties (Table 1).

Table 1. Biodegradable polymers for TEVGs. $T_{\mathrm{m}}$ : melting temperature; $T_{\mathrm{g}}$ : glassforming temperature; Mpa: mega pascal; GPa: giga pascal; P(LA/CL): copolymer of Llactide and $\varepsilon$-caprolactone; PCL: poly( $\varepsilon$-caprolactone); PHB: poly(3-hydroxybutyrate).

\begin{tabular}{ccccccc}
\hline Polymer & $T_{\mathrm{m}}$ & $T_{\mathrm{g}}$ & $\begin{array}{c}\text { Initial } \\
\text { tensile } \\
\text { strength } \\
(\mathrm{Mpa})\end{array}$ & $\begin{array}{c}\text { Elastic } \\
\text { modulus }(\mathrm{GPa})\end{array}$ & $\begin{array}{c}\text { Elongation } \\
\text { at break } \\
(\%)\end{array}$ & $\begin{array}{c}\text { Degradation } \\
\text { period }\end{array}$ \\
\hline PGA & 230 & 36 & 890 & 8.4 & 30 & $2-3$ weeks
\end{tabular}

\begin{tabular}{ccccccc}
\hline PLA & 170 & 56 & 900 & 8.5 & 25 & $6-12$ months \\
\hline $\begin{array}{c}\text { P(LA/CL }) \\
(75: 25)\end{array}$ & 140 & 22 & 500 & 4.8 & 70 & $8-10$ weeks \\
\hline $\begin{array}{c}\text { P(LA/CL) } \\
(50: 50)\end{array}$ & 105 & 17 & 12 & 0.9 & 600 & $4-6$ weeks \\
\hline PCL & 60 & 60 & 50 & 0.3 & 70 & 12 weeks \\
\hline PHB & 177 & 4 & 43 & - & 5 & $>6$ weeks \\
\hline
\end{tabular}

Several biodegradable polymers have been investigated for their suitability in arterial tissue engineering applications. PLA and PCL are commonly used materials for constructing arterial scaffolds due to their successful clinical history [51]. Both PLA and PCL have hydrophobic properties and are maintained within the body for prolonged periods of time. Combining PCL and PLA forms new copolymers called PLCL. Further fine tuning of PLCL scaffold mechanical properties and degradation rates is accomplished by changing molecular weights through composition ratio adjustments. The feasibility of PLA-PLCL scaffolds as small diameter arterial conduits in high pressure environments in vivo has been confirmed [8]. Natural proteins, such as silk fibroin and chitosan, have also been utilized as biodegradable materials for small diameter arterial grafts, and have shown favorable vessel remodeling with long-term patency (Figure 6) [52,53].

\subsubsection{Structural characteristics of arterial TEVGs}

Ideal small diameter arterial TEVGs are; readily available ("off-the-shelf"), biocompatible, easily implanted, capable of transforming into neotissue comparable to that of native arteries, and is resistant to thrombosis, 
aneurysmal dilatation, and ectopic calcification [54]. Biodegradable scaffolds with large pore sizes, fast degradability, and high elasticity are thought to be crucial to obtain the aforementioned TEVG characteristics, as well as promote better and more rapid neotissue formation in small-diameter arterial grafts.

\subsubsection{Sponge type scaffold}

Highly porous, PLCL sponge-type scaffolds, reinforced with PGA mesh, have been successfully applied clinically in low pressure environments [5]. PLCL co-polymer, prepared from $50 \%$ L-lactide and $50 \% \varepsilon$-caprolactone, has high elasticity that is suitable for tissue-engineering applications [55,56]. Even though outer layer reinforcement is required to withstand high arterial pressure, PLCL sponge type scaffolds show great potential and promise as arterial scaffolds due to their elastic properties [57].

While the slow degradation of certain polymers aids in mechanical property retention, allowing grafts to endure high pressures for longer periods of time, it consequently results in undesirable delayed tissue remodeling. Wu et al. were able to demonstrate rapid tissue remodeling using a sponge type scaffold composed of the fast degrading poly(glycerol sebacate) (PGS) elastomer [10], and in a rat arterial implantation model, the PGS scaffold displayed favorable vessel remodeling, in addition to good patency [34].

\subsubsection{Electrospinning technique}

Electrospinning technologies have enabled the production of nanofiber-based scaffolds, and because of their ability to improve cellular infiltration and endothelialization in comparison to standard synthetic grafts [58], have shown promise with regards to arterial scaffold fabrication (Figure 7).
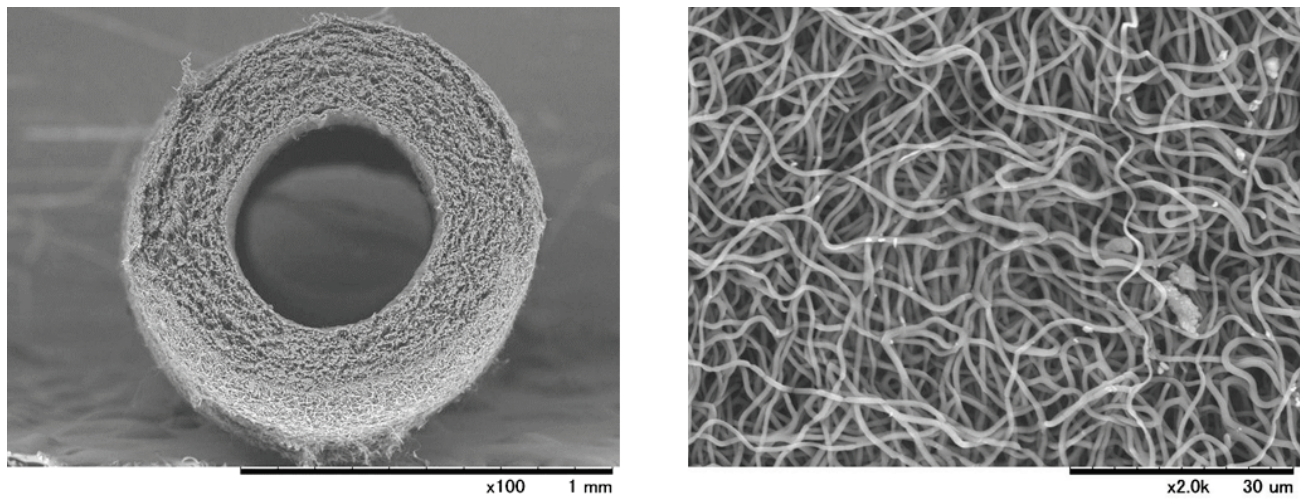

Figure 7. Representative electronic microscope images of poly(lactic acid) nanofiberbased scaffold for aortic graft. Electrospinning technologies have enabled the production of nanofiber-based scaffolds, which have shown promise with regard to arterial scaffold fabrication [54]. 
Small-diameter electrospun scaffolds composed of PCL [59], and PLCL [60], have shown good surgical and mechanical properties with high patency rates in an arterial implantation model. However, small-fiber diameter and tightly knit electrospun scaffolds displayed poor cellular migration into the scaffold, causing prolonged neotissue remodeling and foreign body reactions. On the other hand, electrospun thick-PCL-fiber, with large pore scaffolds enhanced the remodeling process and vascular regeneration by mediating M2 phenotype macrophage polarization [61]. Furthermore, electrospun nanofibers show more promise with their encapsulation and controlled drug release potentials $[60,62]$ that may one day lead to cell-free TEVGs.

\subsection{CONCLUSION}

Arterial TEVGs must be able to withstand high arterial pressure until they are completely reconstituted by host derived cells. Well-designed arterial TEVGs require the formation of well-organized neotissue, characterized by: confluent endothelialization surrounded by SMCs, favorable elastin deposition, and the absence of calcification and stenosis. For the next generation of arterial TEVGs, scaffold improvements will focus on: facilitating cellular infiltration through high porosity and large pore sizes; and fast scaffold degradation in order to enable rapid remodeling, thereby reducing calcification caused by foreign body reactions.

\section{REFERENCES}

1. J.P. Vacanti, R. Langer. Lancet 354 (Suppl 1) (1999) SI32-SI34.

2. R. Langer, J.P. Vacanti. Science. 260 (1993) 920-926.

3. C.B. Weinberg, E. Bell. Science 231 (1986) 397-400.

4. T. Shin'oka, Y. Imai, Y. Ikada. N. Engl. J. Med. 344 (2001) 532-533.

5. G. Matsumura, N. Hibino, Y. Ikada, H. Kurosawa, T. Shin'oka. Biomaterials 24 (2003) 2303-2308.

6. T. Shin'oka, G. Matsumura, N. Hibino, Y. Naito,M. Watanabe, T. Konuma, T. Sakamoto, M. Nagatsu, H. Kurosawa. J. Thorac. Cardiovasc. Surg. 129 (2005) 1330-1338.

7. N. Hibino, E. McGillicuddy, G. Matsumura, Y. Ichihara, Y. Naito, C. Breuer, T. Shinoka. J. Thorac. Cardiovasc. Surg. 139 (2010) 431-6, 436 e1-2.

8. J.D. Roh, G.N. Nelson, M.P. Brennan, T.L. Mirensky, T. Yi, T.F. Hazlett, G. Tellides, A.J. Sinusas, J.S. Pober, W.M. Saltzman, T.R. Kyriakides, C.K. Breuer. Biomaterials 29 (2008) 1454-1463.

9. T.L. Mirensky, G.N. Nelson, M.P. Brennan, J.D. Roh, N. Hibino, T. Yi, T. Shinoka, C.K. Breuer. J. Pediatr. Surg. 44 (2009) 1127-1132; discussion 1132-1133.

10. W. Wu, R.A. Allen, Y. Wang. Nat. Med. 18 (2012) 1148-1153.

11. F. Kuwabara, Y. Narita, A. Yamawaki-Ogata, M. Satake, H. Kaneko, H. Oshima, A. Usui, Y. Ueda. J. Artif. Organs 15 (2012) 399-405. 
12. J.D. Roh, R. Sawh-Martinez, M.P. Brennan, S.M. Jay, L. Devine, D.A. Rao, T. Yi, T.L. Mirensky, A. Nalbandian, B. Udelsman, N. Hibino, T. Shinoka, W.M. Saltzman, E. Snyder, T.R. Kyriakides, J.S. Pober, C.K. Breuer. Proc. Natl. Acad. Sci. U.S.A. 107 (2010) 4669-4674.

13. Y. Naito, T. Shinoka, D. Duncan, N. Hibino, D. Solomon, M. Cleary, A. Rathore, C. Fein, S. Church, C. Breuer. Adv. Drug. Deliv. Rev. 63 (2011) 312-323.

14. A.W. Clowes, T.R. Kirkman, M.A. Reidy. Am. J. Pathol. 123 (1986) 220-230.

15. J.G. Meinhart, M. Deutsch, T. Fischlein, N. Howanietz, A. Froschl, P. Zilla. Ann. Thorac. Surg. 71 (2001) S327-S331.

16. X. Yue, B. van der Lei, J.M. Schakenraad, G.H. van Oene, J.H. Kuit, J. Feijen, C.R. Wildevuur. Surgery 103 (1988) 206-212.

17. L.E. Niklason, J. Gao, W.M. Abbott, K.K. Hirschi, S. Houser, R. Marini, R. Langer. Science 284 (1999) 489-493.

18. M. Peck, D. Gebhart, N. Dusserre, T.N. McAllister, N. L'Heureux. Cells Tissues Organs. 195 (2012) 144-158.

19. A. Muto, T.N. Fitzgerald, J.M. Pimiento, S.P. Maloney, D. Teso, J.J. Paszkowiak, T.S. Westvik, F.A. Kudo, T. Nishibe, A. Dardik. J. Vasc. Surg. 45 (Suppl A) (2007) A15-A24.

20. G.K. Owens. Physiol. Rev. 75 (1995) 487-517.

21. A. Ratcliffe. Matrix Biol. 19 (2000) 353-357.

22. L. Buttafoco, N.G. Kolkman, P. Engbers-Buijtenhuijs, A.A. Poot, P.J. Dijkstra, I. Vermes, J. Feijen. Biomaterials 27 (2006) 724-734.

23. A. Patel, B. Fine, M. Sandig, K. Mequanint. Cardiovasc Res. 71 (2006) 40-49.

24. L. Venkataraman, A. Ramamurthi. Tissue Eng. Part A 17 (2011) 2879-2889.

25. S. Tara, H. Kurobe, M.W. Maxfield, K.A. Rocco, T. Yi, Y. Naito, C.K. Breuer, T. Shinoka. J. Vasc. Surg. 62 (2015) 734-743.

26. J. Hu, P.E. Van den Steen, Q.X. Sang, G. Opdenakker. Nat. Rev. Drug. Discov. 6 (2007) 480-498.

27. J.A. Jones, A.K. McNall, D.T. Chang, L.A. Qin, H. Meyerson, E. Colton, I.L. Kwon, T. Matsuda, J.M. Anderson. J. Biomed. Mater. Res. A 84 (2008) 158-166.

28. J.P. Habashi, D.P. Judge, T.M. Holm, R.D. Cohn, B.L. Loeys, T.K. Cooper, L. Myer, E.C. Klein, G. Liu, C. Calvi, M. Podowski, E.R. Neptune, M.K. Halushka, D. Bedja, K. Gabrielson, D.B. Rifkin, L. Carta, F. Ramirez, D.L. Huso, H.C. Dietz. Science 312 (2006) 117-121.

29. B.S. Brooke, J.P. Habashi, D.P. Judge, N. Patel, B. Loeys, H.C. Dietz. N. Engl. J. Med. 358 (2008) 2787-2795.

30. S. de Valence, J.C. Tille, D. Mugnai, W. Mrowczynski, R. Gurny, M. Moller, B.H. Walpoth. Biomaterials 33 (2012) 38-47.

31. M.Y. Speer, H.Y. Yang, T. Brabb, E. Leaf, A. Look, W.L. Lin, A. Frutkin, D. Dichek, C.M. Giachelli. Circ. Res. 104 (2009) 733-741.

32. Y. Sun, C.H. Byon, K. Yuan, J. Chen, X. Mao, J.M. Heath, A. Javed, K. Zhang, P.G. Anderson, Y. Chen. Circ. Res. 111 (2012) 543-552.

33. S. Tara, H. Kurobe, K.A. Rocco, M.W. Maxfield, C.A. Best, T. Yi, Y. Naito, C.K. Breuer, T. Shinoka. Atherosclerosis 237 (2014) 684-691.

34. R.A. Allen, W. Wu, M. Yao, D. Dutta, X. Duan, T.N. Bachman, H.C. Champion, D.B. Stolz, A.M. Robertson, K. Kim, J.S. Isenberg, Y. Wang. Biomaterials 35 (2014) 165-173. 
35. N. Hibino, T. Yi, D.R. Duncan, A. Rathore, E. Dean, Y. Naito, A. Dardik, T. Kyriakides, J. Madri, J.S. Pober, T. Shinoka, C.K. Breuer. FASEB J. 25 (2011) 4253-4263.

36. Y. Naito, M. Williams-Fritze, D.R. Duncan, S.N. Church, N. Hibino, J.A. Madri, J.D. Humphrey, T. Shinoka, C.K. Breuer. Cells Tissues Organs. 195 (2012) 60-72.

37. P.Y. Chen, L. Qin, C. Barnes, K. Charisse, T. Yi, X. Zhang, R. Ali, P.P. Medina, J. Yu, F.J. Slack, D.G. Anderson, V. Kotelianski, F. Wang, G. Tellides, M. Simons. Cell Rep. 2 (2012) 1684-1696.

38. B.C. Cooley, J. Nevado, J. Mellad, D. Yang, C. St Hilaire, A. Negro, F. Fang, G. Chen, H. San, A.D. Walts, R.L. Schwartzbeck, B. Taylor, J.D. Lanzer, A. Wragg, A. Elagha, L.E. Beltran, C. Berry, R. Feil, R. Virmani, E. Ladich, J.C. Kovacic, M. Boehm. Sci. Transl. Med. 6 (2014) 227ra34.

39. D.R. Duncan, P.Y. Chen, J.T. Patterson, Y.U. Lee, N. Hibino, M. Cleary, Y. Naito, T. Yi, T. Gilliland, H. Kurobe, S.N. Church, T. Shinoka, T.M. Fahmy, M. Simons, C.K. Breuer. J. Am. Coll. Cardiol. 65 (2015) 512-514.

40. A. Kitao, Y. Sato, S. Sawada-Kitamura, K. Harada, M. Sasaki, H. Morikawa, S. Shiomi, M. Honda, O. Matsui, Y. Nakanuma. Am. J. Pathol. 175 (2009) 616-626.

41. D. Medici, S. Potenta, R. Kalluri. Biochem. J. 437 (2011) 515-520.

42. G. Matsumura, S. Miyagawa-Tomita, T. Shin'oka, Y. Ikada, H. Kurosawa. Circulation 108 (2003) 1729-1734.

43. N. Hibino, G. Villalona, N. Pietris, D.R. Duncan, A. Schoffner, J.D. Roh, T. Yi, L.W. Dobrucki, D. Mejias, R. Sawh-Martinez, J.K. Harrington, A. Sinusas, D.S. Krause, T. Kyriakides, W.M. Saltzman, J.S. Pober, T. Shin'oka, C.K. Breuer. FASEB J. 25 (2011) 2731-2739.

44. T. Pennel, P. Zilla, D. Bezuidenhout. J. Vasc. Surg. 58 (2013) 1053-1061.

45. T. Asahara, T. Murohara, A. Sullivan, M. Silver, R. van der Zee, T. Li, B. Witzenbichler, G. Schatteman, J.M. Isner. Science 275 (1997) 964-967.

46. T. Asahara, H. Masuda, T. Takahashi, C. Kalka, C. Pastore, M. Silver, M. Kearne, M. Magner, J.M. Isner. Circ. Res. 85 (1999) 221-228.

47. S. Kaushal, G.E. Amiel, K.J. Guleserian, O.M. Shapira, T. Perry, F.W. Sutherland, E. Rabkin, A.M. Moran, F.J. Schoen, A. Atala, S. Soker, J. Bischoff, J.E. Mayer, Jr. Nat. Med. 7 (2001) 1035-40.

48. M. Sata. Trends Cardiovasc. Med. 13 (2003) 249-253.

49. J. Yu, A. Wang, Z. Tang, J. Henry, B. Li-Ping Lee, Y. Zhu, F. Yuan, F. Huang, S. Li. Biomaterials 33 (2012) 8062-8074.

50. K.C. Rustad, M. Sorkin, B. Levi, M.T. Longaker, G.C. Gurtner. Organogenesis 6 (2010) 151-157.

51. K.A. Athanasiou, G.G. Niederauer, C.M. Agrawal.Biomaterials 17 (1996) 93-102.

52. S. Enomoto, M. Sumi, K. Kajimoto, Y. Nakazawa, R. Takahashi, C. Takabayashi, A. Asakura, M. Sata. J. Vasc. Surg. 51 (2010) 155-164.

53. M. Zhou, W. Qiao, Z. Liu, T. Shang, T. Qiao, C. Mao, C. Liu. Tissue Eng. Part A 20(1-2) (2013) 79-91.

54. S. Tara, K.A. Rocco, N. Hibino, T. Sugiura, H. Kurobe, C.K. Breuer, T. Shinoka. Circ J. 78 (2014) 12-19.

55. A.G. Mikos, Y. Bao, L.G. Cima, D.E. Ingber, J.P. Vacanti, R. Langer. J. Biomed. Mater. Res. 27 (1993) 183-189. 
56. S.I. Jeong, B.S. Kim, Y.M. Lee, K.J. Ihn, S.H. Kim, Y.H. Kim. Biomacromolecules 5 (2004) 1303-1309.

57. S.H. Kim, E. Chung, S.H. Kim, Y. Jung, Y.H. Kim, S.H. Kim. J. Biomater. Sci. Polym. Ed. 21 (2010) 289-302.

58. H. Wu, J. Fan, C.C. Chu, J. Wu. J. Mater. Sci. Mater. Med. 21 (2010) 3207-3215.

59. E. Pektok, B. Nottelet, J.C. Tille, R. Gurny, A. Kalangos, M. Moeller, B.H. Walpoth. Circulation 118 (2008) 2563-2570.

60. S. Wang, X.M. Mo, B.J. Jiang, C.J. Gao, H.S. Wang, Y.G. Zhuang, L.J. Qiu. Int. J. Nanomedicine 8 (2013) 2131-2139.

61. Z. Wang, Y. Cui, J. Wang, X. Yang, Y. Wu, K. Wang, X. Gao, D. Li, Y. Li, X.L. Zheng, Y. Zhu, D. Kong, Q. Zhao. Biomaterials 35 (2014) 5700-5710.

62. Y. Su, Q. Su, W. Liu, G. Jin, X. Mo, S. Ramakrishna. J. Biomater. Sci. Polym. Ed. 23(7) (2012) 861-871. 


\section{Chapter 9}

